\title{
Improved Artificial Fish Swarm Algorithm and its Application in System Identification
}

\author{
Junlin Zhu, Hui Liu and Zulin Wang \\ College of Electrical Engineering and Automation \\ Jiangxi University of Science and Technology \\ Ganzhou, Jiangxi, China \\ e-mail: besterfulitu@163.com
}

Keywords: Artificial Fish Swarm Algorithm, Needle-in-haystack Problem, Parameter Identification

\begin{abstract}
In order to solve the traditional identification method limitations, This paper presents an improved artificial fish swarm algorithm, Through the experiment of a typical Needle-in-haystack problem, Show that the improved artificial fish swarm algorithm has better ability of global optimization, faster convergence speed, higher accuracy of optimization. This algorithm is applied to the system parameter identification, Through to the linear system and nonlinear system parameter identification simulation, Results show that the algorithm has fast convergence, high accuracy advantages, Has important application value in Engineering.
\end{abstract}

\section{Introduction}

System identification is a theory and methods used to study the establishment of the mathematical model of the production process. Traditional system identification methods, although the development of more mature and perfect, but there are still many problems. Such as: system identification based on least squares method generally requires the input signal must have known and more changes; for a linear system with better identification results, but for nonlinear systems are often not satisfied with the results of identification; can not be together to determine the structure and parameters of the system, and often can not get the global optimal solution[1-4].

Artificial Fish Swarm Algorithm was put forward as a bionic optimization algorithm by Doctor. Xiaolei Li in China in 2002[5, 6].The algorithm has good ability to overcome local optima, to obtain the global extremum, and it has flexible use in practice with high convergence velocity, especially in non-linear and strong coupling system fields.

\section{Artificial Fish Swarm Algorithm Principle and Improvement Measures}

In this paper, some improvements were made for individual foraging behavior of artificial fish. Set the current state of artificial fish individual is $X_{i}=\left(X_{i 1}, X_{i 2}, \ldots X_{i n}\right)$. Within the scope of their perceptions, randomly select a state $X_{j}=\left(X_{j 1}, X_{j 2}, \ldots X_{j n}\right)$, if the objective function $Y_{j}<Y_{i}$, then in this direction to move a step forward; conversely, again randomly selected state $\mathrm{X}_{\mathrm{j}}$, try Try_number times, if still do not meet the conditions to go forward, Then random moving step.

Improved pseudo code are described below:

$$
\begin{aligned}
& \text { float Artificial_fish::AF_prey() } \\
& \{ \\
& \text { for } \quad(i=; i<\text { Try_number } ; i++) \\
& X_{j 1}=X_{i 1}+(2 * \text { Rand()-1)•Visual, } \\
& X_{j 2}=X_{i 2}+(2 * \text { Rand()-1)•Visual } \text {; } \\
& X_{j n}=X_{i n}+(2 * \text { Rand()-1)•Visual } ; \\
& \text { if }\left(Y_{j}<Y_{i}\right)
\end{aligned}
$$




$$
\begin{aligned}
& X_{i \mid \text { next }}=X_{i}+\text { Rand }() \cdot \text { Step } \cdot \frac{X_{j}-X_{i}}{\| X_{j}-X_{i} / /} ; \\
& \text { else } \\
& X_{i \mid \text { next }}=X_{i 1}+(2 * \text { Rand }()-1) \cdot \text { Step } ; \\
& X_{i 2 \mid \text { next }}=X_{i 2}+(2 * \text { Rand }()-1) \cdot \text { Step } ; \\
& \ldots \ldots \\
& X_{\text {in|next }}=X_{i n}+(2 * \text { Rand }()-1) \cdot \text { Step } ; \\
& \} \quad \\
& \text { Return AF foodconsistence }\left(X_{i} \mid \text { next }\right) ;
\end{aligned}
$$

\section{Test the Improved Artificial Fish Swarm Algorithm}

In order to verify the improved artificial fish swarm algorithm optimization performance,to test for a typical needle in a haystack problem.

$$
\max f(x, y)=\left(\frac{3.0}{0.05+\left(x^{2}+y^{2}\right)}\right)^{2}+\left(x^{2}+y^{2}\right)^{2}
$$

Where $\mathrm{x}, \mathrm{y} \in[-5.12,5.12]$.

The global maximum value of the function : $\operatorname{maxf}(0,0)$ is 3600.4 local maxima points are: $(-5.12,5.12),(-5.12,-5.12),(5.12,5.12),(5.12,-5.12)$, function value is 2748.78 . Solving using the MATLAB programming,parameter is set to:Visual is 16 ;initial value Step is 0.5 ; artificial fish swarm scale $N$ is 80 ; Try_number is 200 ; $\delta$ is 0.9 ; Friend_number is 30 ; number of iterations $m$ is 800; 2 consecutive simulation results such as shown in Table 1 and Figure 1, 2.

Table 12 consecutive simulation results

\begin{tabular}{|c|c|c|c|}
\hline $\begin{array}{c}\text { Test serial } \\
\text { number }\end{array}$ & $\mathrm{X}$ & $\mathrm{Y}$ & $\operatorname{maxf}(\mathrm{x}, \mathrm{y})$ \\
\hline 1 & $0.278840010378834 \times 10^{-8}$ & $-0.129072784259521 \times 10^{-8}$ & $3.59999999999999998 \times 10^{3}$ \\
\hline 2 & $0.125614296650442 \times 10^{-8}$ & $0.006031639137663 \times 10^{-8}$ & 3600 \\
\hline
\end{tabular}

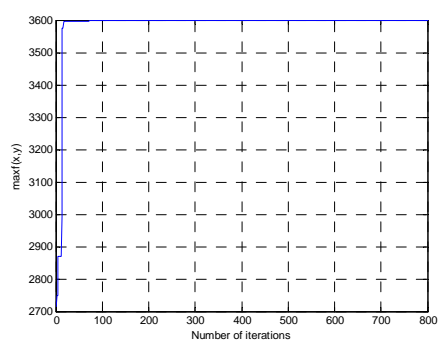

Fig. 1 Objective function optimization process in the first simulation

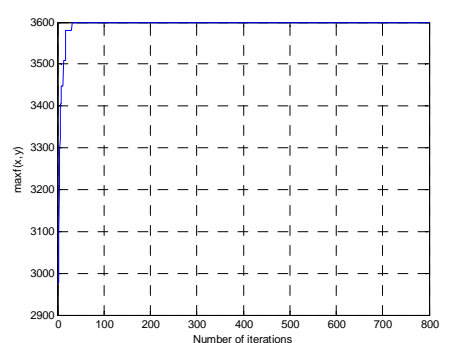

Fig. 2 Objective function optimization process in the Second simulation

Through the simulation found: Initial value of the biggest moving step of artificial fish to be set up appropriate, too small too easy to fall into local optimum; with the increase in the number of iterations, the biggest moving step to reduce gradually, this can improve the solution accuracy. Simulation results show that, Improved artificial fish swarm algorithm is a good solution in needle in a haystack problem, can well out of local extreme value point, faster search to global extreme value point, solution accuracy is high.

\section{Improved Artificial Fish Swarm Algorithm Applied to the System Parameter Identification}

Ideas of Improved Artificial Fish Swarm Algorithm for Parameter Identification. The system parameter identification is through the system simulation, calculate the model output $Y_{j}$, in the same with the actual system disturbances, and then compare the model output $Y_{j}$ and the actual output $\mathrm{Y}_{\mathrm{i}}$ difference between the two, constitute the objective function, to constantly correct the 
unknown parameters of the mathematical model, thereby minimize the objective function value. Use of improved artificial fish swarm algorithm to find the optimal solution in the feasible solution set of unknown parameters, minimize the objective function value, this is the basic idea of improved artificial fish swarm algorithm applied to the parameter identification[7].

Selection of the Objective Function of Improved Artificial Fish Swarm Algorithm. Set $Y_{i}$ is the actual system response output sequence, $Y_{j}$ is the response output sequence of the same input of the mathematical model of the system. Selection of the system response and model output response output sequence sum of square error as the objective function. The objective function is:

$$
f=\sum C\left(Y_{i}-Y_{j}\right)^{2}
$$

Where $\mathrm{C}$ is the normal number.

\section{Simulation Experiment.}

Simulation of Parameter Identification of Three Order Linear System. Identification object is a three order linear system, the transfer function as follows:

$$
G(s)=\frac{k}{a_{1} s^{3}+a_{2} s^{2}+a_{3} s+1}
$$

On the system parameters $\left[\mathrm{k}, \mathrm{a}_{1}, \mathrm{a}_{2}, \mathrm{a}_{3}\right]$ identification, using the improved artificial fish swarm algorithm,the exact value should be $[10,6,11,6]$. The first use of traditional identification method, presumably determine the parameters $\left[\mathrm{k}, \mathrm{a}_{1}, \mathrm{a}_{2}, \mathrm{a}_{3}\right]$ search range,is $[0,20]$. Improved artificial fish swarm algorithm parameter is set to:Visual is 16 ;initial value Step is 5; artificial fish swarm scale $N$ is 50; Try_number is 50; $\delta$ is 1 ; Friend_number is 10 ; number of iterations $m$ is 80 ;

When the simulation, with the increase in the number of iterations, the biggest moving step to reduce gradually. The objective function of the parameter $\mathrm{C}$ is set to 10 . Under the conditions of the unit step input, the sampling period is set to $0.5 \mathrm{~s}$, the simulation time is set to $30 \mathrm{~s}$. Parameter identification process shown in Figure 4.

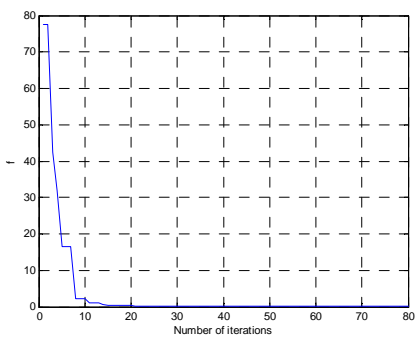

Fig. 4 the identification process of the objective function $\mathrm{f}$

The identification results are as follows: the objective function $\mathrm{f}$ is $6.042036228057711 \times 10^{-8}$; parameter $\mathrm{k}$ is 10.000011942044759 ; parameter $\mathrm{a}_{1}$ is 6.000370439759505 ; parameter $\mathrm{a}_{2}$ is 11.000040875597294 ; parameter $\mathrm{a}_{3}$ is 6.000021059032874 .

Identification results show that, the convergence speed of the algorithm is fast in linear system identification, identification precision is high.

Nonlinear System Identification Simulation. For nonlinear system identification,the use of the traditional identification algorithm, it is hard to find a better solution,but the use of this method can effectively overcome this problem. Below to identify the model parameters of the nonlinear system , through improved artificial fish swarm algorithm. The system transfer function is as follows:

$$
G(s)=\frac{k e^{-\tau s}}{a_{1} s^{3}+a_{2} s^{2}+a_{3} s+1}
$$

The parameters set of identification are $\left[k, a_{1}, a_{2}, a_{3}, \tau\right]$,the exact value should be $[10,6,11,6,3]$. The first use of traditional identification method, presumably determine the parameters $\left[k, a_{1}, a_{2}, a_{3}\right]$ search range, is $[0,20]$, the search area of $\tau$ is $[0,10]$. Improved artificial fish swarm algorithm 
parameter is set to:Visual is 16 ;initial value Step is 5; artificial fish swarm scale $N$ is 60 ; Try_number is $30 ; \delta$ is 0.9 ; Friend_number is 20 ; number of iterations $m$ is 80 ;

When the simulation,with the increase in the number of iterations, the biggest moving step to reduce gradually. The objective function of the parameter $\mathrm{C}$ is set to 10 . Under the conditions of the unit step input, the sampling period is set to $0.2 \mathrm{~s}$, the simulation time is set to $30 \mathrm{~s}$. Parameter identification process shown in Figure 5.

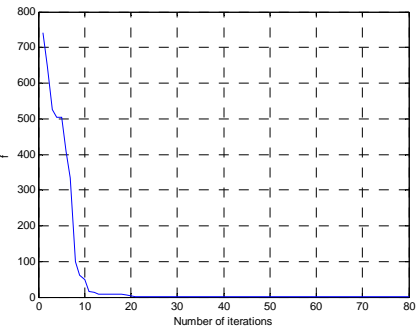

Fig. 5 the identification process of the objective function $\mathrm{f}$

The identification results are as follows: the objective function $\mathrm{f}$ is $7.280276675701756 \times 10^{-5}$; parameter $\mathrm{k}$ is 10.000051674686786 ; parameter $\mathrm{a}_{1}$ is 5.989339987906452 ; parameter $\mathrm{a}_{2}$ is 10.991989494400919; parameter $\mathrm{a}_{3}$ is 5.998908847372268; parameter $\tau$ is 3.000988154905970 .

Identification results show that, the convergence speed of the algorithm is also fast in nonlinear system identification, identification precision is also high.

\section{Conclusion}

This paper presents an improved artificial fish swarm algorithm. The algorithm in global optimization and fast convergence capability, as well as search accuracy has greatly improved. The algorithm is applied in the linear system and nonlinear system parameters identification on the effect is very good. In order to solve the limitation of traditional identification method provides an effective way.

\section{References}

[1] Lin Wang, Ping Ma: Information on Electric Power, Vol.4(2001)No.(4),p.63.

[2] Runqiang Bian, Zengqiang Chen and Zhuzhi Yuan: Control and Decision, Vol.15(2000) No.(5),p.623.

[3] Shuan Liu, Fei Tang: Systems Engineering - Theory and Practice, Vol.27(2007)No.(3),p.134.

[4] Xiaoping Xu,Fucai Qian and Ding Liu: Journal of System Simulation, Vol.20(2008)No. (13),p.3525.

[5] Xiaolei Li: A New Intelligent Optimization Method-Artificial Fish School Algorithm(MS. Zhe Jiang University Control Science and Engineering ,china 2003),p.18.

[6] Xiaolei Li, Zhijiang Shao and Jixin Qian: Systems Engineering Theory and Practice, Vol.22 (2002) No.(11),p.32.

[7] Ziwu Ren,Ye San:Journal of System Simulation, Vol.18(2006) No.(1),p.41. 